\title{
MOBILE HEALTH MONITORING SYSTEM USING FUZZY LOGIC
}

\author{
Naseem Rao \\ Research Scholar, ECE Deptt. Al-Falah University, Faridabad, Haryana, India \\ Anil Kumar \\ Vice Chancellor, Al-Falah University, Faridabad, Haryana, India \\ T. A. Abbasi \\ Adjunct Professor, ECE Deptt. Al-Falah University, Faridabad, Haryana, India
}

Abstract

This paper was tested on a set of data in order to prove its effectiveness. Simulation gives promising results in detecting the activity of the person. This is efficient system for health monitoring as compare with conventional system. In this paper, to identify person's activity fuzzy logic is used. It is found that this system is quick, easy to handle and robust in health monitoring.

\section{Index Terms}

Fuzzy, Health Monitoring, Membership Functions.

\section{INTRODUCTION}

Novel wireless, ambulatory, real-time, and auto alarm intelligent tele cardiology system to improve healthcare for cardiovascular disease proposed by Chin-Teng Lin et.al [1]. Shihab A. Hameed, Vladimir Miho et.al have built a prototype system using open source technologies for centralized medical patient records that can be viewed and updated by physicians using any web browser[2]. M.F.A Rasid, and B. Woodward et.al [3] focus on the design of a processor, which samples signals from sensors on the patient. Yuo et.al proposed wireless sensor network based e-health system based on radio-active and radio-passive positioning [4]. U. Anliker, J. A. Ward et.al has developed a wearable medical monitoring and alert system aimed at people at risk from heart and respiratory diseases [5]. Fuzzy logic can gather performance and intelligibility and it deals with imprecision and uncertainty. It has a background application history to clinical problems including use in automated diagnosis [6], control systems [7], image processing [8] and pattern recognition [9].

E-mental health is frequently used to refer to internet based interventions and support for mental health conditions. However, it can also refer to the use of information and communication technologies that also includes the use of social media, landline and mobile phones. E-mental health services can include information; peer support services, computer and internet based programs, virtual applications and games as well as real time interaction with trained clinicians. Programs can also be delivered using telephones and interactive voice response (IVR)

E-mental health has a number of advantages such as being low cost, easily accessible and providing anonymity to users. However, there are also a number of disadvantages such as concerns regarding treatment credibility, user privacy and confidentiality. Online security involves the implementation of appropriate safeguards to protect user privacy and confidentiality. This includes appropriate collection and handling of user data, the protection of data from unauthorized access and modification and the safe storage of data

In this paper, we propose a fuzzy platform which measures the vital health parameters of the patient or person and also monitors the daily activity to provide pro-active health. As shown in figure 1 e-health monitoring systems are robust, unique and effective. FL requires some numerical parameters in order to operate such as what is considered significant error and significant rate-of-change-of-error, but exact values of these numbers are usually not critical unless very responsive performance is required in which case empirical tuning would determine them. For example, a simple temperature control system could use a single temperature feedback sensor whose data is subtracted from the command signal to compute "error" and then time-differentiated to yield the error slope or rate-of-change-of-error, hereafter called "error-dot". Error might have units of degs $\mathrm{F}$ and a small error considered to be $2 \mathrm{~F}$ while a large error is 5F. The "error-dot" might then have units of degs/min with a small error-dot being $5 \mathrm{~F} / \mathrm{min}$ and a large one being $15 \mathrm{~F} / \mathrm{min}$. These values don't have to be symmetrical and can be "tweaked" once the system is operating in order to optimize performance. Generally, $\mathrm{FL}$ is so forgiving that the system will probably work the first time without any tweaking.

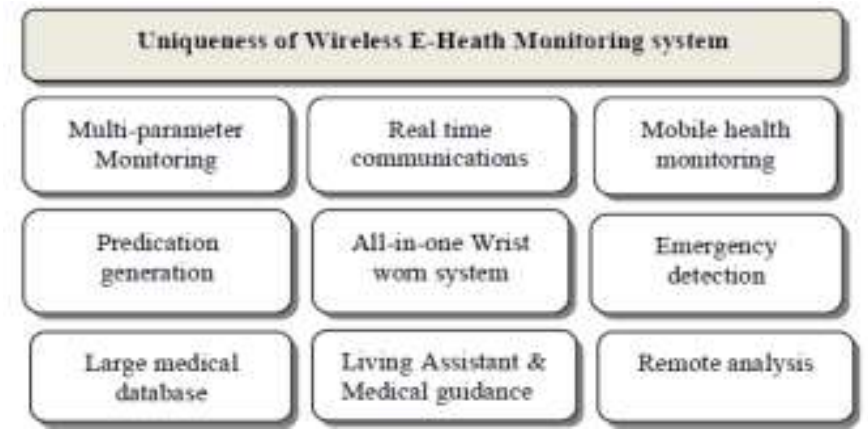

Figure 1. Unique features of wireless e-heath monitoring system 


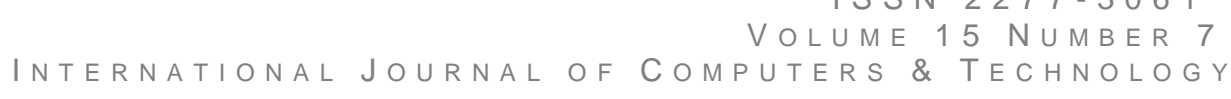

Figure 2 shows the general purpose Fuzzy Logic System which is used in health monitoring of a patient or person on need basis.

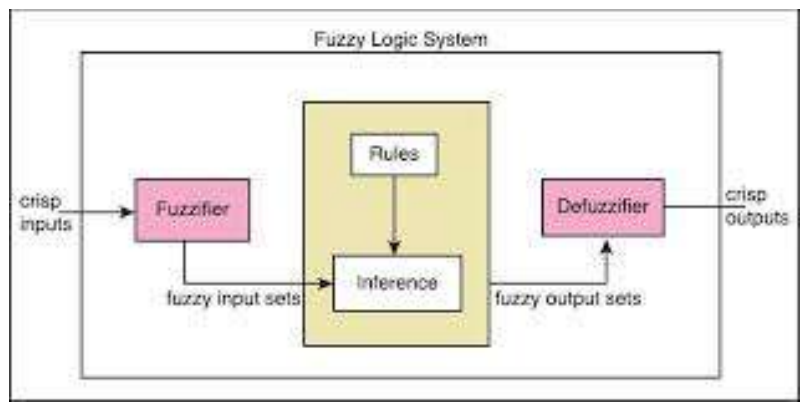

Figure 2: Fuzzy Logic System

Figure 3 shows the FIS file in FIS editor, displaying 5 input functions, Rules and Output function.

\section{HOW IS FL DIFFERENT FROM CONVENTIONAL CONTROL METHODS?}

Fuzzy Logic incorporates a simple, rule-based IF X AND Y THEN Z approach to a solving control problem rather than attempting to model a system mathematically. The FL model is empirically-based, relying on an operator's experience rather than their technical understanding of the system. For example, rather than dealing with temperature control in terms such as "SP $=500 \mathrm{~F}$ ", "T $<1000 \mathrm{~F}$ ", or "210C <TEMP $<220 \mathrm{C} "$, terms like "IF (process is too cool) AND (process is getting colder) THEN (add heat to the process)" or "IF (process is too hot) AND (process is heating rapidly) THEN (cool the process quickly)" are used. These terms are imprecise and yet very descriptive of what must actually happen. Consider what you do in the shower if the temperature is too cold: you will make the water comfortable very quickly with little trouble. FL is capable of mimicking this type of behavior but at very high rate.

Our approach based on fuzzy logic provides robust and high accuracy recognition system of the activity of the person. It gives the output in terms of degree of associativity of the activity with Normal and Ab - normal category. Thus, it helps in co-relating the activity output with both the categories.

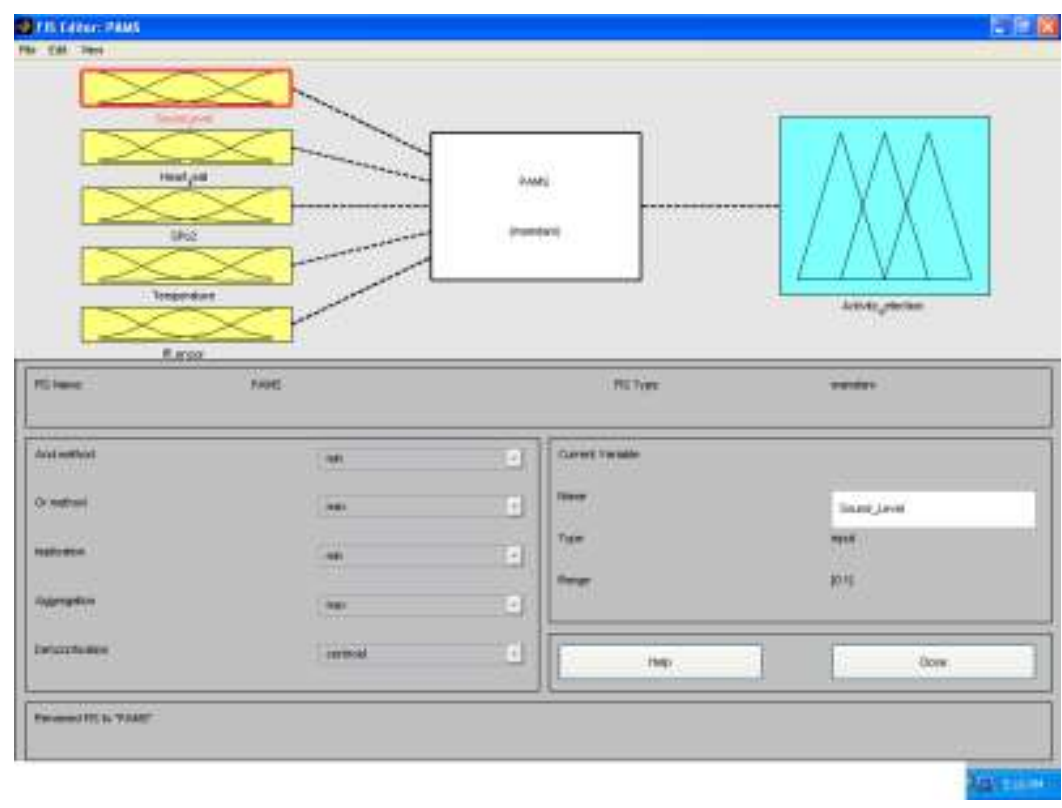

Figure 3: Input and Output of fuzzy system

As compared to conventional system which gives output on fixed boundary i.e. whether a person is Normal or Abnormal, by using fuzzy logic based system, if a person health tends to Detroit, the system will give an indication in the early stage. With increase in values of abnormal parameters, the DOA value will tend to increase more towards abnormal condition as compared to Normal condition, which may help in early detection of person's abnormality.

We have tested this approach on real time data and this system proves to be more efficient as compared to conventional system. In real time testing, we used Trapazoidal membership functions for output within a range of 0 to 10 for Normal and abnormal conditions. For normal condition, we specified the membership function range as [ $\left[\begin{array}{llll}0 & 0 & 3\end{array}\right]$ and for abnormal condition the range is defined as [3 710 10]. So, if the output of the system comes out to be a value 4, then it categories the person in both output categories: Normal with $\mathrm{DOA}=.7$ and for Ab normal, DOA comes out to 0.3. While in conventional system, output will either be Normal or Abnormal. Figure 4 shows the output membership function. 


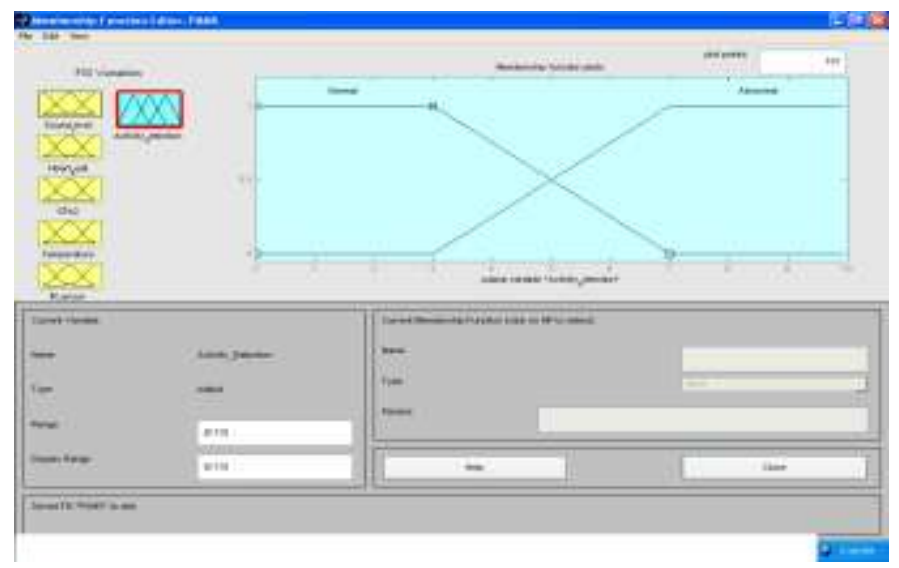

Figure 4 below shows the membership function of output variable defined in FIS file.

\section{Conclusion}

This paper was experimentally tested on a set of data in order to prove its effectiveness. This simulation gave very promising results in detecting the activity of the person. This is efficient system for health monitoring as compare with conventional system. Our approach based on fuzzy logic provides robust and high accuracy recognition system of the activity of the person. It gives the output in terms of degree of associativity of the activity with Normal and Ab - normal category.

\section{References}

[1] Chin-Teng Lin, Fellow, Kuan-Cheng Chang, Chun-Ling Lin, Chia-Cheng Chiang, Shao-Wei Lu, Shih-Sheng Chang, Bor-Shyh Lin, Hsin-Yueh Liang, Ray-Jade Chen, Yuan-Teh Lee, and Li-Wei Ko, "An Intelligent Telecardiology System Using a Wearable and Wireless ECG to Detect Atrial Fibrillation" Member, IEEE Transaction on information technology in biomedicine, Vol. 14, No. 3, May 2010.

[2] Shihab A. Hameed, Vladimir Miho," Medical, Healthcare, and Emergency Model", International Conference on Computer and Communication Engineering (ICCCE 2010), 11-13 May 2010, Kuala Lumpur, Malaysia.

[3] M.F.A Rasid, and B. Woodward, "Bluetooth telemedicine processor for multichannel biomedical monitoring via mobile cellular networks," IEEE Transaction on information technology in biomedicine, vol. 9, no. 1, pp. 35- 43, March 2005.

[4] U. Anliker, J. A. Ward, P. Lukowicz, G. Tröster, F. Dolveck, M. Baer, F. Keita, E. Schenker, F. Catarasi, and R. Schmid, "AMON: A Wearable multi parameter medical monitoring and alerts system", IEEE Trans. On Inf. Technol., Biomed., vol. 8, no. 4, pp. 415-427, 2004.

[5] Hairong Yan, Hongwei Huo, "Wireless Sensor Network Based E-Health System- Implementation and Experimental Results", Member, IEEE Trans. On Consumer Electronics, vol. 56, no. 4, November 2010.

[6] P. Bonato, "Wearable sensors/systems and their impact on biomedical engineering," IEEE Eng. Med. Biol. Mag., vol. 22, no. 3, pp. 18-20, May/Jun. 2003.

[7] L.A. Zadeh, "Fuzzy sets as a basis for theory of possibility, Fuzzy Set Systems," pp. 3-28, 1978.

[8] M. Sugeno, "Theory of fuzzy integrals and its applications," Doct. Thesis, Tokyo Institute of technology 1974.

[9] G. Shafer, "A Mathematical Theory of Evidence," Princeton Univ. Press 1979.

[10] J. C. Bezdek and S. K. Pal, "Fuzzy Models for Pattern Recognition," IEEE Press, 1992.

[11] M. Mizumoto, J. Toyoda and K. Tanaka, "General formulation of formal grammars," Info Sci., 4:87-100, 1972.

[12] Y. H. Pao, "Adaptive Pattern Recognition and Neural Networks," Addison-Wesley, 1989. 